\title{
Memorias, subjetividades y actores sociales
}

\author{
Memories, subjectivities, and social actors
}

Olga Marlene Sánchez Moncada ${ }^{1}$

\section{Resumen}

En este artículo se presenta una síntesis temática a modo de balance sobre la producción desarrollada en cinco años de la Maestría en Estudios Sociales de la Universidad Pedagógica Nacional. De hecho, los estudiantes de la línea "Memoria, identidades y actores sociales" han adelantado sus respectivas investigaciones en torno a estas temáticas con sus correspondientes especificidades. En cuanto a las memorias, han trabajado indagaciones sobre ciencias sociales escolares y la configuración de memoria nacional, en las cuales se han acompañado procesos de construcción de memoria con organizaciones sociales, además de analizar procesos de construcción de memorias sobre el conflicto armado colombiano. Asimismo, se ha abordado una variedad de temas en el enfoque dado a los individuos con potencial de cambio y transformación social. Finalmente, en relación con las subjetividades se han explorado temas como: performatividad, identidades atribuidas, identidades autoatribuidas y la constitución de sujeto.

Palabras clave:

Memoria, identidad, subjetividad, actores sociales.

Abstract

This paper offers a thematic synthesis of the research developed over the five years of the Master's Program in Social Studies at the National Pedagogical University in Bogota. Indeed, students pursuing the emphasis on "Memories, identities and social actors" have developed their research around these issues following their own specificities. Regarding memories, they have worked some issues about school Social Sciences and nationwide collective memory patterns. With respect to this last issue, some students have worked alongside with social organizations in order to analyze processes of memory building about the Colombian armed conflict. Also, a range of topics have been approached to study individuals with potential for social change and transformation. Finally, in relation to subjectivities, topics such as performativity, ascribed identities, self-ascribed identities, and the constitution of the subject have been explored.

Keywords:

Memories, identity, subjectivity, social actors.

1 Docente Departamento de Ciencias Sociales, coordinadora de la línea Memorias, subjetividades y actores sociales de la Maestría en Estudios Sociales de la Universidad Pedagógica Nacional. Bogotá. Colombia. Correo electrónico: osanchez@ pedagogica.edu.co 


\section{Trabajos con la memoria}

Los trabajos con la memoria se han desplegado en tres ámbitos: las ciencias sociales escolares y la configuración de memoria, los procesos de construcción de memoria con organizaciones sociales y construcción de memorias sobre el conflicto armado colombiano.

\section{Configuración de la memoria nacional y ciencias sociales escolares}

En aquella articulación entre los problemas nacionales y la escuela, las ciencias sociales escolares empiezan a incorporar reflexiones de todo tipo acerca de la presencia y/o ausencia sobre la construcción de memoria, en particular de la memoria nacional.

En este sentido, en el trabajo "Configuración de la memoria nacional en el discurso educativo de las ciencias sociales escolares en Colombia 1976-2004" (Aponte Otálvaro, 2012) se confronta el argumento que considera que las memorias fuertes logran este estatuto gracias a la fortaleza del aparato estatal o a la imposición unidireccional de los medios tecnológicos de información. Asumida la memoria como narrativa o construcción social discursiva, en esta investigación se hallaron estrategias de fijación de la memoria nacional que conducen a la configuración de estereotipos del ser colombiano, formación de estereotipos sociales y producción de una memoria nacional a partir de ciertos rasgos de la población colombiana.

En el discurso educativo de la historia escolar del periodo estudiado, también se identificaron aquellas marcas de la memoria nacional, se analizó la producción discursiva de emblemas patrios realizada en las asignaturas de instrucción cívica, educación para la democracia y educación constitucional y se exploró la formación del sentimiento patrio en las alusiones a los hechos heroicos y ejemplos de gestas en la formación de la nación.

\section{Construcción de políticas de la memoria de las organizaciones sociales}

Además de lo escolar, las organizaciones sociales es otro de los ámbitos de análisis en la pregunta por la memoria en un país que se dice sin memoria. En trabajo conjunto con la Organización Femenina Popular [OFP], se estudió el proceso de configuración de las políticas de la memoria de esta organización y su incidencia en la identidad política de sus integrantes. Estas políticas se abordaron en tres niveles: político, simbólico e histórico; apartir del rastreo de sus marcas territoriales y conmemoraciones. La identidad política se trabajó desde dos perspectivas, el principio de distinguibilidad y la configuración arquetípica y estereotípica que la organización construye simbólicamente (Alfonso Gil, 2011, p. 27).

Esta relación entre marcos de configuración de políticas de la memoria e identidad política ocurre en el devenir histórico y político de la región del Magdalena Medio a partir de 1972 (Alfonso Gil, 2011, p. 2); por lo cual en este estudio se da cuenta del contexto histórico del entorno sociopolítico de la OFP y de sus condiciones de emergencia a partir de la historia de Barrancabermeja como enclave petrolero, epicentro de las luchas sindicales y políticas, y como escenario de las misiones jesuíticas.

Se presentan las reivindicaciones y luchas políticas de la organización, apartir de las marcas territoriales de la memoria representadas en la Casa de la Mujer; las conmemoraciones de fechas emblemáticas; y las movilizaciones que la organización adelanta periódicamente en defensa de sus derechos. También se analiza la producción iconográfica desde la perspectiva de la configuración del mito en el ámbito político y de la construcción de consignas que caracterizan las demandas de la OFP; con base en autores como Jelin, Barthes y Deleuze, respectivamente (Alfonso Gil, 2011, p. 2).

En esta línea de análisis que hace énfasis en la construcción de memoria con las organizaciones sociales, se encuentran en curso las siguientes investigaciones ${ }^{2}$ :

- Prácticas de memoria en las organizaciones sociales.

2 Rosario Arias Callejas, Luis Giovanni Rodríguez Castillo, Magda Marleny Cárdenas Suárez, Luvin Hernando Camargo Umbarila y Rafael Antonio Morales, respectivamente. 
- La conmemoración del día del orgullo gay en la última década en Bogotá.

- Acción colectiva de las organizaciones sociales trans en Bogotá en la configuración/potenciación de sus identidades: aportes a la recuperación de la memoria del movimiento trans.

- Memoria del trabajo realizado por Orlando Fals Borda con los campesinos de la vereda de Saucío-Chocontá (Cundinamarca).

- Memorias de las prácticas deportivas en el municipio de Fómeque (Cundinamarca).

\section{Construcción de memoria y conflicto armado}

Los ámbitos académicos, políticos, culturales y de las organizaciones sociales, son algunos de los diferentes lugares desde los cuales se construye memoria sobre el conflicto armado colombiano, lugares que podrían corresponder a los campos de emergencia de las luchas políticas por las memorias de este conflicto.

Estas luchas políticas se expresan en los procesos de construcción de memoria que se vienen consolidando en varias partes del país, en donde han ocurrido diversidad de acontecimientos violentos como las masacres y todo tipo de hechos considerados como crímenes de lesa humanidad. Uno de estos hechos, es la "masacre de Trujillo", asumida como una práctica sistemática y constante de violación a los derechos humanos, efectuada por agentes del Estado, grupos de narcotraficantes y de paramilitares a la población de los municipios de Trujillo, Riofrío y Bolívar (Valle), durante los años 1986 y 1994.

En estas poblaciones organizadas, confluyen proyectos e iniciativas de memoria especialmente promovidas por las organizaciones sociales como la Comisión Intercongregacional de Justicia y Paz [CIJP], en conjunto con la Asociación de Familiares Víctimas de Trujillo [AFAVIT] (Martínez Mora, 2010, p. 58).

En el 2005 por efecto de la Ley de Justicia y Paz se crea la Comisión Nacional de Reparación y Reconciliación [CNRR] y con ella la Comisión de
Memoria Histórica [CMH], esta entidad oficial será en adelante la encargada de la reconstrucción de memoria histórica de algunos casos emblemáticos, esta nominación se refiere a masacres que, en sus componentes, contienen elementos susceptibles de ser tipificados. El objetivo político de estas acciones de memoria está en función de la verdad, la justicia, la reparación y la reconciliación, bajo el presupuesto de que Colombia se encuentra en postconflicto.

El primer caso trabajado por la CNRR correspondió a la masacre de Trujillo, asumido como caso emblemático. Se definen como casos emblemáticos aquellos "lugares de condensación de procesos múltiples que se distinguen no sólo por la naturaleza de los hechos, sino también por su fuerza explicativa" (CNRR, 2008, p. 20)3. La construcción de memoria desde este lugar y desde las organizaciones sociales, de derechos humanos y víctimas de la masacre de Trujillo, fueron el objeto de análisis para indagar por los siguientes aspectos: los referentes políticos, criterios de elección y categorizaciones que aparecen en estas superficies de emergencia en relación con la memoria, los emisores y emprendedores en la construcción de memoria de la masacre de Trujillo, sus formas organizativas y sus ejes de controversia, entre otros asuntos.

En este campo de investigación sobre la memoria y el conflicto armado, se encuentran en curso las investigaciones ${ }^{4}$ :

3 Después del informe sobre Trujillo, la Comisión de Memoria Histórica o actual Grupo de Memoria Histórica ha trabajado los siguientes casos y publicado los siguientes informes: “Trujillo: Una tragedia que no cesa" (2008), “La masacre de El Salado: esa guerra no era nuestra" (2009), “La Rochela, memorias de un crimen contra la justicia” (2010), “Bojayá: La guerra sin límites” (2010), “La masacre de Bahía Portete: Mujeres Wayuu en la mira" (2010), “La tierra en disputa. Memorias del despojo y resistencias campesinas. 19602010" (2010), “Silenciar la democracia. Las masacres de Remedio y Segovia" (2010), "La masacre de El tigre Putumayo" (2011), “El orden desarmado. La resistencia de la Asociación de trabajadores campesinos del Carare (ATCC)" (2011), “Desplazamiento forzado en la comuna 13: la huella invisible de la guerra" (2011), "San Carlos: memorias del éxodo en la guerra" (2011), “Mujeres y guerra: Víctimas y resistentes en el Caribe Colombiano" (2011), "Mujeres que hacen historia. Tierra, cuerpo y política en el Caribe colombiano" (2011), "El Placer. Mujeres, coca y guerra en el Bajo Putumayo" (2012), “Nuestra vida ha sido nuestra lucha. Resistencia y memoria en el Cauca indígena" (2012), entre otros.

4 José Luis Blanco Núñez y Jhon Castiblanco Campos, respectivamente. 
- Reelaboración de la memoria de los integrantes de las organizaciones sociales del Catatumbo acerca de las luchas sociopolíticas desarrolladas antes y después de la incursión paramilitar de 1999.

- La masacre de jóvenes en el suroriente de Bogotá.

\section{Trabajo con los actores sociales}

Los actores sociales motivo de indagación por parte de los estudiantes de la línea han sido asumidos como sujetos potencia de cambio y transformación; en la actualidad se cuenta con tres trabajos concluidos, uno con el resguardo de Ambaló (Silvia, Cauca), otro con las madres comunitarias de Ciudad Bolívar y Usme, y el último con los trabajadores y las trabajadoras que han venido luchado por la defensa del Hospital San Juan de Dios como un bien público; a su vez, en el campo de los actores sociales contamos con varios proyectos en curso.

El primer trabajo corresponde al primer estudiante egresado de la línea "Memoria, identidades y actores sociales", quien desarrolló el estudio "Proyecto político indígena y propuesta educativa comunitaria. El caso del resguardo de Ambaló, Silvia (Cauca)", en el que analizó las prácticas escolares del sistema de educación propia del Consejo Regional de Indígena del cauca (CRIC y su relación con la política comunitaria (Jaime, 2010).

A partir de los planteamientos de Hugo Zemelman -en particular de las categorías necesidad política, utopía, y experiencia- se indagó por el componente político del proyecto educativo y su política comunitaria, los proyectos que permiten su desarrollo, su impacto en el tejido social y su relación con el plan de vida; mediante el uso de la etnografía con entrevistas abiertas y observación participante, las historias de vida, la minga de pensamiento y consulta documental.

El objetivo principal fue comprender las prácticas comunitarias que permiten la concreción del proyecto político, dinamizadas desde los proyectos pedagógicos del Centro Educativo de Agoyan de la comunidad de Ambaló. Estos proyectos son de carácter cultural, ambiental, productivos, organizativos, entre otros; resaltando los aportes de cada uno de estos al proyecto político.

Con esta investigación se logra construir una propuesta de análisis para los procesos políticos de los diferentes movimientos sociales, en la que no se naturalizan los procesos indígenas sino que se abordan en medio de interacciones, identificando rompimientos, fortalecimientos, recuperaciones, adopciones y creaciones de realidad, y proponiendo que cada uno de estos aspectos pueden ser analizados en los diferentes proyectos políticos.

Por su parte, las Madres Comunitarias surgen en los años ochenta, principalmente en barrios que recién se venían configurando en los extramuros de la ciudad o barrios llamados populares con alta población infantil. La investigación "El papel de las mujeres en los barrios populares de Bogotá a través del cuidado de la infancia: historia de vida madres comunitarias y jardineras 1980-2011 en las localidades de Ciudad Bolívar y Usme". (García Ortiz, 2012, p. 2-3), indaga por su presencia en el tiempo, su papel fundamental en el desarrollo de sus barrios y su perfilamiento como líderes y actores sociales dentro de sus comunidades y da cuenta de cómo la organización y el establecimiento de solidaridades para estas mujeres fueron mecanismos clave para sus iniciativas en el cuidado de niños y niñas.

El estudio se realizó desde un enfoque etnográfico para posicionar la voz de las mujeres sujeto, en el entendido de que actúan en estructuras de desigualdad social y cultural que les impide pronunciarse sobre temas de los que ellas son protagonistas. Para cumplir con este objetivo metodológico se trabajó con la historia de vida de cada una de ellas. (García Ortiz, 2012, p. 3)

En relación con la investigación sobre el Hospital San Juan de Dios, el propósito fue establecer las consecuencias sociales y laborales del proceso de liquidación del hospital, particularmente desde el punto de vista de los trabajadores y las trabajadoras que han luchado por la defensa de éste como un bien público. Se realizó un acercamiento a la historia de 
la institución, ratificando su importancia cultural y patrimonial en el desarrollo de la medicina en Colombia, en la enseñanza y en la profesionalización de las ciencias de la salud.

También se reconstruyó su proceso de liquidación en el marco de la reforma del sector salud y la lucha que por su defensa como institución pública han desarrollado las enfermeras y trabajadores del mismo, junto con las implicaciones laborales y sociales del proceso de liquidación del Hospital San Juan de Dios. Igualmente, la investigación describe la vida cotidiana (profesional, laboral, familiar) de las enfermeras y trabajadores del hospital que aún asisten cotidianamente, a pesar de su liquidación, en la perspectiva de recuperar la memoria colectiva y la identidad que de este proceso tienen sus protagonistas. El trabajo en mención dio lugar a caracterizar la situación del Hospital San Juan de Dios como emblemática de la crisis de la red hospitalaria pública frente al proceso de reforma del sector salud. (Granada Osorio, 2012, p. 32)

Además de la comunidad del resguardo de Ambaló, las madres comunitarias, y de las trabajadoras y trabajadores del Hospital San Juan de Dios, en la actualidad se adelantan las siguientes investigaciones sobre actores sociales, constituyéndose en un campo de creciente interés:

- Los movimientos sociales y educación en América latina: el caso de los bachilleratos populares en Buenos Aires.

- Los nuevos sujetos en la línea de base del MIRA.

- Sentido de lo público y la construcción de un "mundo común" en el movimiento estudiantil colombiano 1971-2013.

- Sistematización de la experiencia de la Casa de derechos de Engativá.

- La autonomía política y los procesos de organización en la localidad de Suba.

5 Diana Paola Triana Moreno, Flor Azucena Rocha Padilla, Magali Smith Pinilla Talero, Liliana Camargo Urrea, Gustavo Eduardo Gaona García, Nicolás Martínez Gómez, Wilder Andrey Tellez González, Diego Andrés Santos Rubiano, Sergio Esteban Segura Guiza y Angélica Johanna Díaz Pomar, respectivamente.
- Discursos y prácticas de la educación popular: La experiencia del colectivo "Tejiendo" en la localidad de Engativá (2010-2013).

- Historia de Evaristo Bernate: praxis en educación popular y teología de la liberación.

- Espacios distorsionados y discos nunca vendidos.

- Construcción de poder popular en los colectivos anarquistas de la Red Libertaria Popular Mateo Krammer.

- Análisis crítico del discurso de las políticas públicas en la construcción de la noción de universidad pública en Colombia: 1991-2011.

\section{Trabajos con subjetividades}

Desde las múltiples discusiones teóricas sobre lassubjetividades, en la línea "Memoria, identidades y actores sociales" se han desarrollado trabajos con diversos énfasis: performatividades, identidades atribuidas, identidades autoatribuidas y la constitución de sujeto, lo cual veremos a continuación.

\section{Análisis sobre la performatividad}

En la investigación "Performatividad y masculinidad en las telenovelas: El capo, Las muñecas de la mafia y Las detectivas y el Víctor", se analizan los procesos de producción de sujetos desde las apuestas de interpelación agenciadas a través de estas telenovelas; para lo cual se cruzaron dos niveles de análisis, los lugares de identificación que proponen las telenovelas y los procesos de encuentro y disputas que agencian las y los televidentes (Vargas Rojas, 2010).

Con base en los planteamientos de Judith Butler y su "Teoría de la performatividad", se asume que los sujetos son construcciones precarias y temporales, definidos en relación con marcos normativos reguladores que intentan construir las posibilidades materiales de los mismos sujetos que después intenta reglamentar. Sin embargo, en ese proceso de reiteración del poder para producir y delimitar a los sujetos, se hace evidente su inestabilidad y temporalidad. Desde este lugar teórico, en esta 
investigación se indaga por los lugares de masculinidades legibles y naturalizadas por los que se apuesta socialmente y su relación con los lugares de constitución de feminidades. Para su desarrollo se examinaron los guiones de las tres telenovelas, sus imágenes promocionales y se realizaron entrevistas semiestructuradas a los televidentes consumidores de este producto televisivo.

Por otra parte, desde la performatividad corporal se adelanta la investigación "Caracterización de las estéticas corporales en el deporte de alto rendimiento. Análisis de contenido fotográfico de Juegos Olímpicos de Verano, 2000-20086".

\section{Identidades en la constitución de sujeto}

En la pregunta por la constitución del sujeto, en particular por lo masculino y femenino del sujeto, se llevó a cabo el trabajo "Crímenes pasionales en Bogotá, 1900-1930” (Castro López, 2011), en el cual se indaga por el victimario y victimaria de estos crímenes, a partir del análisis que desde lo jurídico y la psiquiatría se produce sobre este tipo de delito. Se observan varios casos ocurridos en Bogotá, entre 1900 y 1930, otros casos sucedidos en los años ochenta del mismo siglo, analizados por Myriam Jimeno -quien aporta teóricamente para el análisis de sobre el victimario de este delito- y casos más recientes.

Se analizan algunas de las principales características de los protagonistas del crimen pasional, las particularidades que se atribuyen al criminal pasional desde las ciencias criminales y la psiquiatría, los elementos fundamentales que componen los procesos judiciales llevados a cabo a los criminales pasionales y un análisis del criminal pasional como agente de profilaxis social, mediante la actuación de la justicia y las valoraciones morales y sociales de cada época. (Castro López, 2011)

Los resultados muestran que los procesos judiciales de los crímenes pasionales contienen elementos comunes a otro tipo de homicidio. En los tres periodos analizados se deduce que en estos procesos

6 Clara Lourdes Peña Castro. ha existido una linealidad histórica en la que prevalece el argumento de la ausencia de una intención libre y dolosa por parte del victimario, quien generalmente se encuentra "enajenado mentalmente a causa de la celotipia"; lo anterior conduce a la debilidad jurídica para castigar el crimen pasional y a justificar esta medida de profilaxis social en contra del adulterio, en la que en la mayoría de los casos las víctimas han sido mujeres. (Castro López, 2011)

Desde esta perspectiva de constitución de sujeto, se encuentran en curso "Los clientes de la prostitución femenina en Bogotá", e "Imaginarios sobre adultos con discapacidad cognitiva leve"7.

\section{Identidad como auto atribución}

Una de las características de otro de los campos de indagación, es su énfasis en dar cuenta de la identidad a partir de aquellas características que los individuos se auto atribuyen a sí mismos como identidad. El trabajo "Contando-se en la reinserción: configuración identitaria de jóvenes desvinculados de grupos armados ilegales, a partir de las historias de vida" explora la manera como se configura la identidad de estos jóvenes, con base en las elaboraciones conceptuales que respecto a la identidad han desarrollado Gilberto Giménez y Stuart Hall, en el marco de los estudios sociales en América Latina y los estudios culturales, respectivamente. (Arévalo Mendoza, 2011)

Encuentra la autora que la identidad que emerge es la del desmovilizado, aun cuando el sujeto que menos tiempo llevaba de haber salido del grupo armado, lo había hecho cerca de cinco años atrás. Por una parte, se asumen como víctimas, lo cual sugiere un aspecto performativo en la construcción identitaria en la medida en que ante determinadas condiciones de tiempo y espacio, los sujetos encuentran en ella la posibilidad de agenciarse. Por otra parte, construyen su red primaria en contextos sociales y culturales distintos a los de su procedencia y los elementos del nuevo contexto son incorporados para recrear dicha identidad. La autora, además,

7 Gustavo Octavio García Rodríguez y Gloria Elsa Rodríguez Jiménez, respectivamente. 
plantea como reto indagar por la configuración de la identidad en el proceso de inserción social en el contexto de latencia del conflicto armado. (Arévalo Mendoza, 2011, p.77)

Otro de los trabajos desde esta perspectiva es "Volta do mundo. La Capoeira Angola en el contexto bogotano: su apropiación y resignificación” (Chavarro Bermeo, 2012), en el que se muestra una aproximación histórica sobre esta práctica, algunas tendencias desde las cuales ha sido abordada como objeto de estudio en las ciencias sociales y se presenta una cartografía que incluye componentes como la roda y los entrenos, la congregación, la musicalidad, algunos rituales internos y prácticas corporales asumidas como prácticas de subjetivación y de reterritorialización.

\section{Políticas de la etnicidad}

Los trabajos agrupados bajo la nominación políticas de la etnicidad, indagan por la identidad atribuida y autoatribuida en las comunidades indígenas. El estudio concluido a la fecha es "La diáspora de los Inganos residentes en la zona céntrica de la ciudad de Bogotá: análisis de las actuales prácticas de identidad y memoria" (Moreno Rubiano, 2013), en el cual se indaga por la relación entre la condición de dispersión del lugar de origen en los inganos con sus procesos actuales de configuración de las prácticas de identidad y memoria en la ciudad de Bogotá.

En este sentido, se exploró: ¿Quién es y cómo se identifica un inga urbano en la ciudad de Bogotá?, ¿cómo las movilizaciones que han realizado los inganos desde su lugar de origen han influido hasta ahora en sus dinámicas de configuración de sus prácticas como pueblo indígena en la ciudad?, ¿qué implicaciones han tenido las movilizaciones que los inganos realizaron desde su lugar de origen con sus procesos actuales de configuración de las prácticas de identidad y memoria en Bogotá?, ¿cómo se realiza la configuración de las prácticas de identidad y la producción de memoria de los inganos que se movilizaron de sus lugares de origen y permanecen en la zona céntrica de la ciudad de Bogotá?
Al respecto se halló que los inganos mantienen vínculo estrecho con el lugar de origen, ya sea real o imaginario; la relación que establecen estas comunidades con la sociedades receptoras no está exenta de conflicto; su asentamiento lleva más de una generación y suceden procesos de hibridación cultural mediante la consolidación de identificaciones en relación con el lugar de origen y con la sociedad receptora, entre otros aspectos. (Moreno Rubiano, 2013, p. 5)

En este campo de las políticas de la etnicidad se encuentran en curso las siguientes investigaciones: "El niño y la niña en las políticas de etnicidad", "Etnicidad y religiosidad en el pueblo inga del Valle de Sibundoy", "Nukanchipaalpaiuiai: territorio, tiempo y espacio en los inga de la Bota Caucana", $\mathrm{y}$ "Pacientes, usuarios y practicantes de medicinas tradicionales y alternativas en Bogotá".

Para cerrar este balance cabe mencionar que se empiezan a configurar estudios sobre temas y problemas de la historia reciente colombiana como el conflicto armado, en esta perspectiva se adelantan las siguientes investigaciones:

- El paramilitar como victimario político: contrainsurgencia, limpieza social y despojo.

- Territorio y despojo: imaginarios en víctimas de desplazamiento forzado.

- Seguridad democrática en caricatura ${ }^{9}$.

\section{Referencias}

Alfonso Gil, M. C. (2011). Políticas de la memoria e identidad política en la Organización Femenina Popular [OFP] (Tesis para optar al título de Maestría en Estudios Sociales). Facultad de Humanidades, Universidad Pedagógica Nacional,Bogotá. [Sandra Patricia Rodríguez Ávila, directora.]

Aponte Otálvaro, J. E. (2012). Configuración de la memoria nacional en el discurso educativo de las ciencias sociales escolares en Colombia 1976-2004 (Tesis para optar al título de Maestría en Estudios Sociales).

8 Sindy Campo Perdomo, Oscar Javier Pulido Angel, Leidy Marcela Bravo Osorio y Vanessa Cano Bermúdez, respectivamente.

9 Dilsa Beatriz Hortua Poveda, Oscar Armando Gil Quintero y Jhonny Santiago Rosero Velasco, respectivamente. 
Facultad de Humanidades, Universidad Pedagógica Nacional, Bogotá. [Sandra Patricia Rodríguez Ávila, directora.]

Arévalo Mendoza, H. (2011). Contando-se en la reinserción: configuración identitaria de jóvenes desvinculados de grupos armados ilegales, a partir de las historias de vida (Tesis para optar al título de Maestría en Estudios Sociales). Facultad de Humanidades, Universidad Pedagógica Nacional, Bogotá. [Alfonso Torres Carrillo, director.]

Castro López, O. A. (2011). Crímenes pasionales en Bogotá, 1900-1930 (Tesis para optar al título de Maestría en Estudios Sociales). Facultad de Humanidades, Universidad Pedagógica Nacional, Bogotá. [Marlene Sánchez Moncada, directora.]

Comisión Nacional de Reparación y Reconciliación [CNRR] (2008). Trujillo: una tragedia que no cesa. Bogotá: Editorial Planeta Colombiana S. A.

Chavarro Bermeo, D. M. (2012). "Volta do mundo". La Capoeira Angola en el contexto bogotano: su apropiación y resignificación (Tesis para optar al título de Maestría en Estudios Sociales). Facultad de Humanidades, Universidad Pedagógica Nacional, Bogotá. [Omar Alberto Garzón Chiriví, director.]

García Ortiz, D. L. (2012). El papel de las mujeres en los barrios populares de Bogotá a través del cuidado de la infancia: historia de vida madres comunitarias y jardineras 1980-2011 en las localidades de Ciudad Bolívar y Usme (Tesis para optar al título de Maestría en Estudios Sociales). Facultad de Humanidades, Universidad Pedagógica Nacional, Bogotá. [Pablo Andrés Nieto Ortiz, director.]

Granada Osorio, G. (2012). El conflicto laboral en el Hospital San juan de Dios. El trabajo de campo en un hospital público "a la deriva” (Tesis para optar al título de Maestría en Estudios Sociales). Facultad de humanidades, Universidad Pedagógica Nacional, Bogotá. [Pablo Andrés Nieto Ortiz, director.]

Jaime, J. C. (2010). Proyecto político indígena y propuesta educativa comunitaria. El caso del resguardo de Ambaló, Silvia (Cauca) (Tesis para optar al título de Maestría en Estudios Sociales). Facultad de humanidades, Universidad Pedagógica Nacional, Bogotá. [Alfonso Torres Carrillo, Director.]

Martínez Mora, N. (2010). Luchas políticas de la memoria sobre el conflicto armado colombiano: El caso de la masacre de Trujillo (Tesis para optar al título de Maestría en Estudios Sociales). Facultad de huma- nidades, Universidad Pedagógica Nacional, Bogotá. [Marlene Sánchez Moncada, directora.]

Moreno Rubiano, Z. Z. (2013). La diáspora de los Inganos residentes en la zona céntrica de la ciudad de Bogotá: análisis de las actuales prácticas de identidad y memoria (Tesis para optar al título de Maestría en Estudios Sociales). Facultad de humanidades, Universidad Pedagógica Nacional, Bogotá. [Omar Alberto Garzón Chiriví, director.]

Vargas Rojas, J. A. (2010). Performatividad y masculinidad en las telenovelas: El capo, Las muñecas de la mafia y Las detectivas y el Víctor (Tesis para optar al título de Maestría en Estudios Sociales). Facultad de humanidades, Universidad Pedagógica Nacional, Bogotá. [Marlene Sánchez Moncada, directora.] 